\title{
ESTIMACIÓN DEL IMPACTO DE FACTORES SOCIOECONÓMICOS SOBRE EL EMPLEO INFORMAL EN EL MERCADO DE TRABAJO MEXICANO
}

\section{ESTIMATION OF THE IMPACT OF SOCIOECONOMIC FACTORS ON INFORMAL EMPLOYMENT IN THE MEXICAN LABOR MARKET}

Mayra Yesenia Nava Rubio
Profesor-Investigador en la Facultad de Ciencias Administrativas de la Universidad Autónoma de Baja California (UABC).
Miembro del Sistema Nacional de Investigadores (SNI).
Doctora en Ciencias Económicas.
Mexicali, Baja California, (México).
E-mail: mnava35@uabc.edu.mx ORCID: https://orcid.org/0000-0003-3206-1353

Rogelio Varela Llamas

Profesor-Investigador en la Facultad de Economía y Relaciones Internacionales de la Universidad Autónoma de Baja California (UABC). Miembro del Sistema Nacional de Investigadores (SNI).

Doctor en Economía Industrial y Relaciones Laborales.

Tijuana, Baja California, (México).

E-mail: rvarela@uabc.edu.mx ORCID: https://orcid.org/0000-0002-8608-4537

Recepción: 04/10/2019 Aceptación: 04/12/2019 Publicación: 24/11/2020

Citación sugerida:

Nava, M. Y., y Varela, R. (2020). Estimación del impacto de factores socioeconómicos sobre el empleo informal en el mercado de trabajo mexicano. 3C Empresa. Investigación y pensamiento crítico, 9(4), 31-51. https://doi. org/10.17993/3cemp.2020.090444.31-51 


\section{RESUMEN}

Las variaciones que muestra el empleo informal en el mercado de trabajo mexicano pueden ser explicadas considerando diversos factores de orden social y económico. El interés por estudiar el empleo informal surge a través de la observación de su gran magnitud como proporción de la población empleada, que es aproximadamente del 60\% a nivel nacional para el periodo que abarca del año 2005 al año 2014. Debido a que la informalidad presenta diversas características, o en otras palabras, es heterogénea, la estimación del impacto de los factores socioeconómicos se realiza considerando su clasificación en empleo informal tipo independiente y empleo informal tipo dependiente. A su vez, los factores pertenecen a una visión teórica dualista o institucionalista. La metodología seleccionada para la estimación es un modelo de efectos fijos con datos de panel que permite concluir que los factores socioeconómicos de mayor impacto sobre los niveles de empleo informal en el mercado de trabajo mexicano son la educación de la población y la suficiencia del sector moderno o formal para captar empleo.

\section{PALABRAS CLAVE}

Factores Socioeconómicos, Empleo Informal, Empleo Informal Independiente, Empleo Informal Dependiente, Efectos Fijos, Panel de Datos. 


\section{ABSTRACT}

The variations shown by informal employment in the Mexican labor market can be explained considering various social and economic factors. The interest in studying informal employment arises through the observation of its great magnitude as a proportion of the employed population, which is approximately 60\% nationally for the period from 2005 to 2014. Because informality It has several characteristics, or in other words, it is heterogeneous, the estimation of the impact of socio-economic factors is made considering its classification in informal type independent employment and dependent type informal employment. In turn, the factors belong to a dualistic or institutionalist theoretical vision. The methodology selected for the estimation is a fixed effects model with panel data that allowes us to conclude that the socioeconomic factors with the greatest impact on the levels of informal employment in the Mexican labor market are the education of the population and the sufficiency of the modern sector or formal to capture employment.

\section{KEYWORDS}

Socioeconomic Factors, Informal Employment, Independent Informal Employment, Dependent Informal Employment, Fixed Effects, Data Panel. 


\section{INTRODUCCIÓN}

La informalidad es un tipo de empleo que posee algunas características positivas, como la disminución del desempleo, pero al que también se le han asociado algunas características negativas que contradicen los esfuerzos por lograr mejores condiciones de ocupación, crecimiento y desarrollo. Son estas peculiaridades las que le conceden importancia de análisis en el ámbito académico y justifican el objetivo gubernamental de reducirla.

En la economía mexicana, la informalidad es un fenómeno que ha alcanzado elevadas cifras, muestra de ello es que durante el periodo 2005-2014, en promedio el 58.8\% de la población ocupada estaba inmersa en ella, de acuerdo a cifras del Instituto Nacional de Estadística y Geografía (INEGI). Por su magnitud, es relevante identificar aquellos factores que contribuyen a explicarla desde un escenario macroeconómico y que sean útiles en el diseño de una estrategia que permita disminuirla.

Es importante señalar que no todo el empleo informal exhibe las mismas características. Una clasificación general en términos de la relación laboral que se sostiene comprende dos grandes categorías; informalidad independiente e informalidad dependiente. La diferencia entre ambas radica en que, en el primer caso, no existe una subordinación de carácter laboral, mientras que en el segundo caso sí.

A nivel teórico, dos enfoques proveen la explicación a dicho fenómeno; la visión dual y la institucional. La primera sostiene que la informalidad se relaciona con la estructura del mercado de trabajo y la decisión de ser informal tiene carácter involuntario. Por su parte, el enfoque institucional destaca que la causa de la informalidad se relaciona con el desempeño de las instituciones del Estado, mismas que pueden influir en favor de la elección de un empleo informal, concediéndole un carácter voluntario.

El objetivo del presente trabajo es determinar la influencia que tiene sobre la informalidad laboral dependiente e independiente un conjunto de factores de carácter dual e institucional, en el marco de un modelo econométrico de datos de panel que considera un horizonte temporal del año 2005 al año 2014 
y 24 entidades federativas de las 32 que conforman la geografía nacional. La exclusión de 8 entidades se debe a la falta de información.

\section{DESARROLLO}

Los orígenes sobre el estudio de la informalidad se remontan a los trabajos de Keith Hart, quien estudió el caso de Ghana a principios de los años setenta, destacando el término de sector informal como "un conjunto de actividades económicas que se encuentran fuera de la fuerza laboral organizada" donde plantea que el empleo informal actúa como un amortiguador para quienes se encuentran sin empleo (Hart, 1973).

La OIT define a la economía informal como el conjunto de actividades económicas que se encuentran insuficientemente contempladas por los sistemas formales o en el extremo no lo están. Aun cuando los individuos y empresas operan dentro de la ley, ésta no se aplica o no se cumple ya que la misma fomenta su incumplimiento al ser inadecuada, compleja o al imponer costos excesivos (OIT, 2012).

Algunos estudios sostienen que la informalidad es un trabajo que se realiza sin contar con el amparo del marco legal o institucional (Negrete y Eternod, 2011). Otros subrayan que puede tener significado diferente para distintas personas, pero comúnmente significa cosas "malas" como: trabajadores sin protección, regulación excesiva, baja productividad, competencia desleal, evasión de la autoridad de la ley, pago incompleto o falta de pago de impuestos y un trabajo desde las sombras (Maloney y Saavedra, 2008).

Por otra parte, la informalidad genera efectos positivos y negativos. Entre los efectos positivos, permite abatir el desempleo al convertirse en una salida a la incapacidad del sector formal para generar empleos. En cambio, los efectos negativos afectan directamente a la economía y al bienestar a través de la falta de acceso a los sistemas de seguridad social o los bajos salarios. Para Aguilera y Velázquez (2005) la 
informalidad puede traer consigo implicaciones negativas para el crecimiento económico de un país, ya que impacta sobre la productividad laboral, que es un determinante fundamental de este.

Leal (2014) menciona tres distorsiones inducidas por la informalidad. La primera, en la demanda óptima de trabajo de las empresas informales, esto es, permanecen pequeñas con el objetivo de evadir la detección de su actividad informal. Por lo que la informalidad se asocia a empresas pequeñas con baja productividad y bajo crecimiento. La segunda se refleja en las elecciones de ocupación, para algunos individuos es más atractivo convertirse en empresarios que permanecer como empleados. Por último, la mala asignación de recursos, es decir, a las empresas con baja productividad se les asignan más recursos de los que deberían.

\subsection{INFORMALIDAD LABORAL: ALGUNAS ESTADÍSTICAS}

La evolución de la informalidad laboral en México para el periodo 2005-2014 se muestra en el Gráfico 1, donde se reportan las cifras de la Tasa de Informalidad Laboral (TIL). Durante el periodo referido, en promedio la TIL fue de 58.8\%, lo que representa un elevado porcentaje de población ocupada informal.

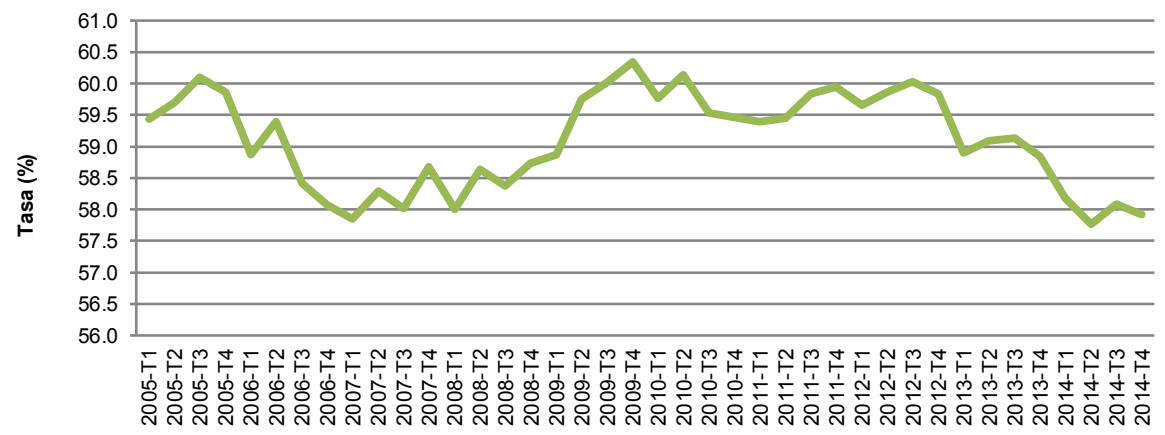

Gráfico 1. Tasa de Informalidad Laboral (TIL) en México.

Fuente: elaboración propia con datos de la Encuesta Nacional de Ocupación y Empleo (ENOE).

A partir del año 2007, la TIL muestra un comportamiento ascendente que alcanza su nivel máximo en el cuarto trimestre de 2009 equivalente a 60.4\%. Esto se relaciona con la contracción que experimentó 
la economía mexicana derivada de los efectos de la crisis internacional suscitada en 2008. Sin embargo, después del evento la tendencia se revierte y al final dicha tasa alcanza niveles muy similares a los registrados en 2007, que son de los más bajos dentro del periodo considerado.

Es conveniente conocer el comportamiento de los componentes de la TIL. En el Gráfico 2, se ilustra la Tasa de Informalidad Laboral Independiente (TILI) con frecuencia anual, esta representa en promedio 21.6\% para el periodo. Del año 2005 a 2008, muestra un comportamiento descendente significativo pasando de $22.4 \%$ a $21.0 \%$, respectivamente. Sin embargo, en 2009 se incrementa a $22.0 \%$ mostrando en lo sucesivo variaciones que distan de su valor más bajo alcanzado en 2008.

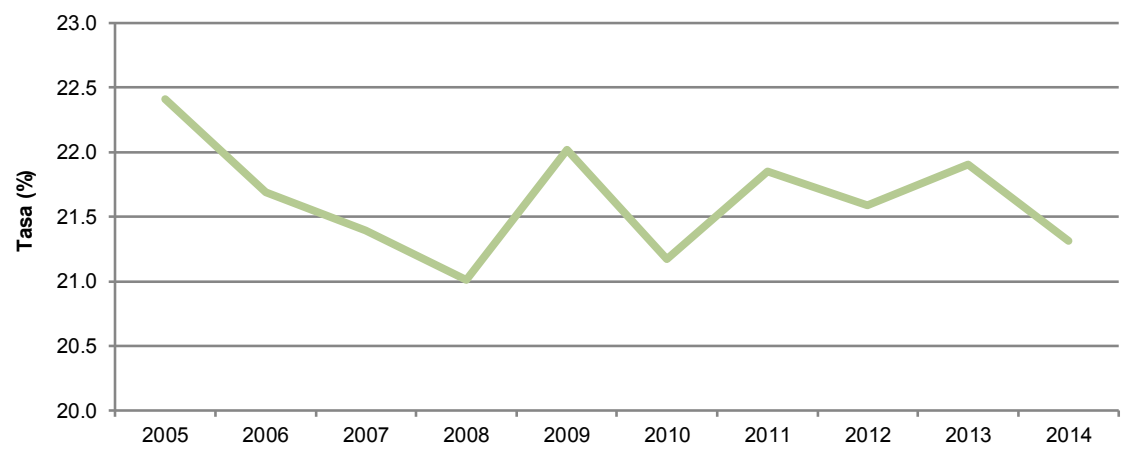

Gráfico 2. Tasa de Informalidad Laboral Independiente (TILI) en México.

Fuente: elaboración propia con datos de la Encuesta Nacional de Ocupación y Empleo (ENOE).

La Tasa de Informalidad Laboral Dependiente (TILD) en el Gráfico 3, en promedio representó $37.2 \%$ para el mismo periodo, siendo superior a la TILI en alrededor de 15.6 puntos porcentuales. En consecuencia, la TIL se compone mayormente por la informalidad del tipo dependiente y es de suponerse que parte de esta se encuentra en el sector formal de la economía. En términos generales, los niveles de informalidad observados en el país resultan elevados, lo que es preocupante desde una visión de calidad del empleo, bienestar social, crecimiento y desarrollo económico. 


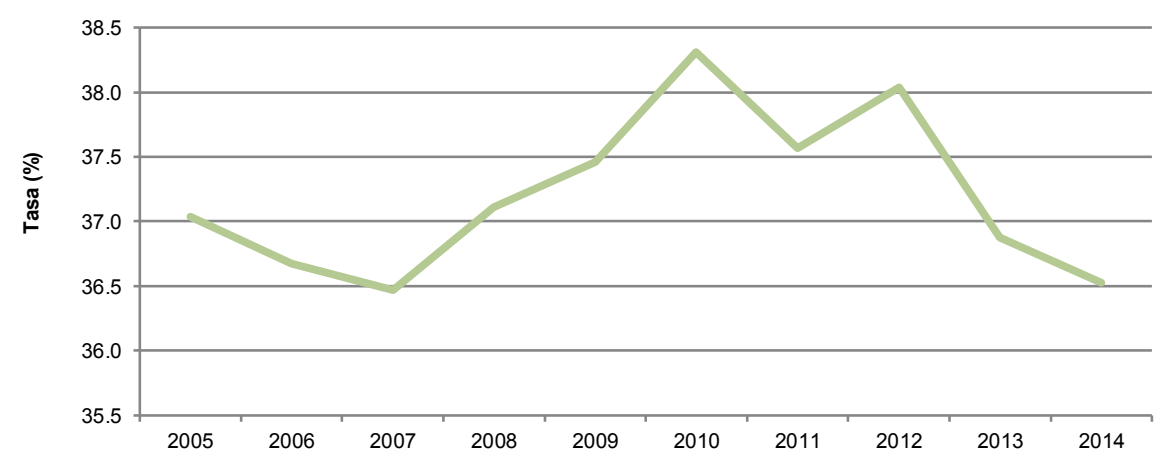

Gráfico 3. Tasa de Informalidad Laboral Dependiente (TILD) en México.

Fuente: elaboración propia con datos de la Encuesta Nacional de Ocupación y Empleo (ENOE).

\subsection{TEORÍA DUAL E INSTITUCIONAL}

Los enfoques teóricos dual e institucional proveen los argumentos fundamentales en la explicación del fenómeno de la informalidad laboral. Comenzando por la teoría dual, para Leontaridi (1998), la dualidad implica que los empleos pertenecen a un sector primario o a uno secundario. A estos sectores se hace referencia con distintas denominaciones, por un lado, un sector llamado "capitalista, formal, moderno, industrial o urbano" y otro considerado "de subsistencia, informal, tradicional, agrícola o rural" (Fields, 2004).

Leontaridi (1998) argumenta que el sector primario comprende a los "buenos" empleos caracterizados por salarios elevados, seguridad económica y rápida rotación que conducen a la promoción profesional. En contraste, el sector secundario comprende a los "malos" empleos que regularmente son no calificados, no ofrecen escalas profesionales y con niveles salariales bajos. Por su parte, Jiménez (2013) sostiene que, para la teoría dualista, los trabajadores informales constituyen la porción excluida del mercado de trabajo formal y la inserción en el mercado informal es involuntaria. 
En Fields (2005) el sector informal es un espacio de libre entrada, es decir, para un individuo es posible obtener un empleo si así lo desea, o las barreras a la entrada son pocas o inexistentes, lo que origina que los procesos de determinación de salarios sean distintos entre el sector formal e informal de la economía.

Una segunda explicación la provee la teoría institucional bajo la cual, las decisiones tomadas desde las instituciones del Estado generan incentivos que influyen en la elección racional de los agentes al escoger entre la ocupación formal o informal (Jiménez, 2012). Es decir, quienes están en el sector informal lo hacen por decisión propia.

Para Uribe y Ortiz (2004), el enfoque institucional ignora las restricciones de la estructura económica, concentrándose en las decisiones que a nivel individual se realizan con respecto a la decisión de participar en la actividad económica. Bajo este enfoque los individuos y empresas escapan al cumplimiento con las instituciones, por ejemplo, evadiendo impuestos. Entonces, el sector informal puede surgir cuando gobierno impone regulaciones e impuestos excesivos, pero carece de la capacidad para hacer cumplir la ley (Loayza, 1997).

Por otro lado, los beneficios que dictan las instituciones que deben recibir los individuos empleados en el sector formal pueden contribuir al incremento de la informalidad, tal es el caso del seguro médico. El empleo del jefe de hogar, en algunos casos ofrece seguro médico para los familiares dependientes principalmente cónyuges e hijos, como resultado, estos últimos pueden obtener relativamente poca rentabilidad de las contribuciones de seguridad social que deberán pagar si se emplean en el sector formal (OCDE, 2004).

\subsection{ANÁLISIS EMPÍRICO EN MÉXICO Y OTROS PAÍSES}

A continuación, se describen los resultados empíricos de algunas investigaciones en torno a la temática de interés. Packard (2007) examina la elección de sector en Chile y modela tres tipos de empleo: un sector formal de empleo asalariado bajo contrato, un sector informal de empleo asalariado sin contrato y el autoempleo. Los resultados indican que el autoempleo no es un sector residual de un mercado laboral 
dualista, es decir, los individuos lo eligen; por el contrario, el empleo informal si exhibe características de un sector residual.

Trabajos como el de Tannuri-Pianto y Pianto (2002) consideran datos de la encuesta de hogares "Pesquisa Nacional por Amostra de Domicílios (PNAD)" para el año de 1999 para Brasil. Estudian a los individuos formales e informales en varios puntos de la distribución de ingresos (por cuantiles) y concluyen que las diferencias más relevantes de las funciones de ingresos son sus recompensas y penalizaciones por cuestiones como el analfabetismo, el género y la afiliación sindical. La penalización es elevada para los individuos analfabetas en el sector formal. Las mujeres son más penalizadas en los cuantiles más altos del sector formal que en el sector informal. Asimismo, ser miembro de un sindicato tiene efecto positivo y significativo sobre los ingresos de los formales, en cambio sólo los trabajadores informales en los cuantiles más altos reciben un beneficio.

Otros trabajos estudian la diferencia salarial entre el sector formal e informal donde se miden las ganancias y pérdidas en los salarios derivados de la formalización. Para el caso de México en el periodo 2000-2003 en Moreno (2007) se reconoce que dos pueden ser las fuentes de la heterogeneidad del salario; la primera es una tasa impositiva aplicada a un sector relativo al otro y la segunda es la autoselección de los agentes entre sectores. Las estimaciones le permiten concluir que la fuente de esa diferencia salarial es la autoselección.

En Friedman et al. (2000), se realiza un estudio de la informalidad desde una perspectiva institucional para 69 países, encontrando que elevadas tasas de impuestos se asocian con menor actividad informal, en cambio la mayor burocracia, mayor corrupción y un entorno legal débil se asocian con una gran economía informal.

Singh et al. (2012) estudian los determinantes de la economía informal con énfasis en las instituciones y el estado de derecho. La unidad de análisis es una muestra de 100 países (avanzados, emergentes y en desarrollo). Los resultados indican que mejores instituciones se asocian a una participación menor de la 
economía informal, y que los países con mayor corrupción tienden a poseer economías informales más grandes.

Para Busso et al. (2012) la excesiva informalidad en México provoca costos en términos de productividad. Considerando los años de 1998 y 2008 los autores argumentan que la informalidad se debe a la regulación asimétrica entre trabajadores asalariados y no asalariados. La regulación laboral establece derechos para los asalariados, así como obligaciones (inscripción al Instituto Mexicano del Seguro Social, pago por despido, salario mínimo, etc.) para las empresas, pero estos no aplican para los no asalariados (informales). Así, la vía por la que afecta la informalidad a la productividad es por las distorsiones creadas en el precio de los factores dada esta asimetría.

\subsection{METODOLOGÍA Y DATOS}

La metodología instrumentada consiste en estimar un modelo de regresión con datos en panel, que considera 24 de las 32 entidades de la federación para el periodo 2005 a 2014. Existen distintas técnicas metodológicas para datos en panel, sin embargo, en el presente caso las pruebas formales como la F-restringida, Multiplicador de Lagrange de Breusch-Pagan y la de Hausman, indican que el modelo adecuado es de efectos fijos. Asimismo, la estimación con variables instrumentales no fue considerada debido a que la prueba de exogeneidad de Sargan, indicó que no existe endogeneidad.

El desarrollo metodológico se realiza siguiendo a Davidson y MacKinnon (1993) quienes parten de un modelo con datos en panel $y_{t i}=x_{t i}(\beta)+u_{t i}$, donde $t$ hace referencia a la dimensión temporal que va desde 1 hasta $T$ periodos, e $i$ es la dimensión espacial o corte transversal que involucra de 1 hasta $n$ unidades de análisis. Por lo tanto, el número total de observaciones es igual a $n T$.

Los datos fueron obtenidos de fuentes como el Banco de Información Económica (BIE) del Instituto Nacional de Estadística y Geografía (INEGI), la Encuesta Mensual de la Industria Manufacturera (EMIM), Encuesta Industrial Mensual (EIM), Encuesta Nacional de Ocupación y Empleo (ENOE), los registros administrativos de las Finanzas Públicas Estatales y Municipales y de otras como el Tercer 
Informe de Gobierno 2014-2015, Consejo Nacional de la Población (CONAPO), Secretaría de Educación Pública (SEP) y el Instituto Nacional para la Evaluación de la Educación (INEE).

El modelo para la informalidad laboral independiente compuesta por los individuos que son trabajadores por cuenta propia y empleadores es $T I L I_{t i}=\alpha_{i}+\beta D_{t i}+\delta I_{t i}+u_{t i}$. El modelo para la informalidad laboral dependiente que registra a individuos que dependen de un jefe y cuyo empleo informal puede ser subordinado y remunerado o no remunerado es $T I L D_{t i}=\alpha_{i}+\beta D_{t i}+\delta I_{t i}+u_{t i}$.

Tres factores integran al vector $D_{t i}$, el Producto Interno Bruto (PIB) pretende mostrar la relación negativa de la situación económica; si es favorable, entonces la informalidad disminuirá, debido a que el sector moderno se encontrará en condiciones para emplear formalmente una cantidad mayor de trabajadores, y viceversa.

El segundo factor es la Educación de la Población Económicamente Activa (PEA) que se expresa como EDU. Si se parte de la consideración de que en el sector informal se aglutinan los empleos menos calificados, entonces entre menor sea el nivel de educación de los trabajadores, el sector informal crecerá. Por consiguiente, la relación entre informalidad y educación se espera negativa.

El tercer factor es el tamaño del Sector Moderno (TSM) medido a través del porcentaje de población ocupada en la industria manufacturera. Se espera una relación negativa, pues la informalidad cambiará de acuerdo con la capacidad del sector moderno para generar empleo. La industria manufacturera es considerada como el sector moderno porque se compone de actividades reconocidas por hacer uso de diversos niveles de tecnología.

Entre los factores que conforman el vector $I_{t i}$ está el Costo Laboral Unitario (COSTO) en el sector moderno. El enfoque institucional resalta que los individuos y empresas buscan escapar a las obligaciones formales, entonces es de esperarse que las empresas analicen el comportamiento del costo laboral al momento de demandar trabajo formal. La relación esperada entre COSTO y la informalidad es 
positiva, a mayores costos laborales mayor informalidad (menor demanda de trabajo formal) y viceversa. El cálculo de COSTO se basó en McConnell et al. (2003).

Para el enfoque institucional las acciones e instituciones del gobierno pueden influir en las variaciones de los niveles de informalidad. Por ello, se involucra en el análisis la eficiencia en recaudación(EFICIENCIA), que mide la proporción de ingresos por impuestos que se gastan en remuneraciones al personal de la Secretaría de Administración y Finanzas y se calcula con base en IMCO (2012).

La relación entre EFICIENCIA e informalidad puede ser positiva o negativa. Es positiva cuando los individuos permanecen en la formalidad dado que perciben que sus contribuciones tributarias mejoran la calidad de las instituciones y obtienen beneficio directo. Es negativa si el individuo considera que esa eficiencia lo perjudica, incrementado el costo de permanecer formal, lo que puede conducirlo a la informalidad.

Un factor adicional es el Seguro Popular (SP), que intenta capturar la decisión voluntaria del individuo por pertenecer a la informalidad. Santana (2011) determina que el bajo costo del SP ocasiona que algunos individuos prefieran permanecer en la informalidad en lugar de afiliarse a un esquema como el del Instituto Mexicano del Seguro Social (IMSS).

En Levy (2008) se refuerza la idea de que los programas de protección social (como el SP) inducen a los trabajadores a tomar un empleo informal. La relación esperada entre SP y la informalidad es positiva, y se calcula como la proporción de la población total que se encuentra afiliada al seguro popular. Si cada vez es mayor el número de individuos que pueden acceder al SP, entonces estos decidirán optar por la informalidad, incrementándose los niveles de esta en el país.

Finalmente, los modelos estimados para la informalidad independiente y dependiente respectivamente son: 


$$
\begin{aligned}
& \text { TILI }_{t i}=\alpha_{i}+\beta_{1} \text { PIB }_{t i}+\beta_{2} \text { EDU }_{t i}+\beta_{3} \text { TSM }_{t i}+ \\
& +\delta_{1} \text { COSTO }_{t i}+\delta_{2} \text { EFICIENCIA }_{t i}+\delta_{3} S_{t i}+u_{t i} \\
& \text { TILI }_{t i}=\alpha_{i}+\beta_{1} \text { PIB }_{t i}+\beta_{2} \text { EDU }_{t i}+\beta_{3} \text { TSM }_{t i}+ \\
& +\delta_{1} \operatorname{COSTO}_{t i}+\delta_{2} \text { EFICIENCIA }_{t i}+\delta_{3} \text { SP }_{t i}+u_{t i}
\end{aligned}
$$

\subsection{RESULTADOS}

La Tabla 1 contiene las elasticidades significativas para el modelo [1]. Cuando se considera la informalidad independiente, sólo dos variables son significativas en su explicación, una corresponde a la teoría dual y otra a la institucional. Esto permite confirmar que los factores de ambos enfoques teóricos influyen en la explicación de esta parte de la informalidad total.

Tabla 1. Modelo [1]: Tasa de Informalidad Laboral Independiente (TILI).

\begin{tabular}{|c|c|c|c|}
\hline \multicolumn{2}{|c|}{ Teoría Dual } & \multicolumn{2}{c|}{ Teoría Institucional } \\
\hline Factor & Elasticidad & Factor & Elasticidad \\
\hline TSM & -0.1345 & EFICIENCIA & -0.0057 \\
\hline
\end{tabular}

Fuente: elaboración propia con base en resultado de estimación.

Se encuentra evidencia que la TILI exhibe una disminución de $0.13 \%$ cuando el factor TSM se incrementa en $1 \%$ en promedio. La informalidad dependiente e independiente se ven afectadas por la dinámica del sector moderno en una magnitud similar. Conforme mayor es la capacidad de absorción de mano de obra formal en el sector moderno, menor es la tasa de informalidad.

Otro resultado es que un incremento de $1 \%$ en promedio en EFICIENCIA, produce un decremento de $0.005 \%$ en la TILI y viceversa. Al incrementarse EFICIENCIA, la informalidad se incrementa como resultado de la búsqueda de los individuos por pagar menos impuestos o evadir los costos de la formalidad. 
En la Tabla 2 se encuentran las elasticidades significativas para TILD. En general, los signos son los esperados y destaca que un mayor número de factores macroeconómicos explican este tipo de informalidad. Desde la perspectiva dual, si el PIB se incrementa en 1\% en promedio la TILD disminuye en $0.04 \%$. Asimismo, un aumento de EDU en 1\% en promedio genera una disminución de la TILD de $0.40 \%$. Este valor es superior al resto de elasticidades e indica que el mayor nivel de educación de los individuos contribuye en gran medida a la disminución de la informalidad dependiente. Por último, el incremento en 1\% en promedio del TSM ocasiona una disminución de $0.13 \%$ de la TILD.

Tabla 2. Modelo [2]: Tasa de Informalidad Laboral Dependiente (TILD).

\begin{tabular}{|c|c|c|c|}
\hline \multicolumn{2}{|c|}{ Teoría Dual } & \multicolumn{2}{c|}{ Teoría Institucional } \\
\hline Factor & Elasticidad & Factor & Elasticidad \\
\hline PIB & -0.0448 & COSTO & 0.0186 \\
\hline EDU & -0.4064 & SP & 0.0476 \\
\hline TSM & -0.1310 & & \\
\hline
\end{tabular}

Fuente: elaboración propia con base en resultado de estimación.

Con relación al enfoque institucional, el primer factor es COSTO cuyo aumento en 1\% en promedio tiene un impacto de $0.01 \%$ en la TILD. Se puede decir que el costo que representan los trabajadores para los empleadores si es un factor que ejerce influencia sobre el número de individuos a contratar como formales; si este costo se incrementa entonces la informalidad responde en el mismo sentido y viceversa.

Por último, SP es un factor cuyo incremento en 1\% en promedio, provoca un aumento de $0.04 \%$ en la TILD, y de forma contraria. Las variaciones en este factor ejercen influencia sobre la informalidad dependiente y puede deberse a que los individuos aceptan un empleo informal en el que saben que la atención médica no será otorgada por el empleador pero que tienen la alternativa de acceder a ella a través del Seguro Popular. 


\section{CONCLUSIONES}

El presente artículo analizó desde una óptica macroeconómica la informalidad laboral en México durante el periodo 2005-2014 de acuerdo a su clasificación en independiente (TILI) y dependiente (TILD), considerando la teoría dual y la institucional planteando un modelo que permite incluirlas, conformando una estructura de datos de panel para 24 de las 32 entidades federativas de México. El modelo fue estimado mediante efectos fijos, robusto a problemas de autocorrelación y heterocedasticidad y descartando problemas de endogeneidad.

Los resultados son acordes a lo esperado, tanto la informalidad independiente como la dependiente responden a las variaciones de factores de carácter dual e institucional, sin embargo, el mayor efecto se observa en los factores de la teoría dual. Específicamente destaca la influencia de TSM sobre la TILI y de EDU sobre la TILD. En términos del valor de las elasticidades, es mayor el de la EDU 0.40 comparado con 0.13 asociada al sector moderno. A partir de estos resultados es posible argumentar que para disminuir la informalidad en el país se deben tomar acciones gubernamentales que mejoren los indicadores macroeconómicos vinculados con la estructura dual del mercado de trabajo, fomentando principalmente la educación de la población. Otra acción es promover el dinamismo del sector moderno de la economía ya que ello permitirá que la absorción de mano de obra se incremente y mejoren las condiciones de bienestar social de la población. Es importante tener en cuenta que el factor TSM es el único que, de acuerdo a las estimaciones, incide significativamente en ambos tipos de informalidad estudiado. Por lo tanto, lograr influir positivamente en este provocará que tienda a reducirse todo tipo de informalidad en el país.

El PIB es un factor estadísticamente significativo, pero únicamente para la informalidad dependiente, lo que sugiere que una situación económica difícil incidirá en un incremento de la informalidad, debido a un efecto contractivo en el mercado laboral expresado en una mayor tasa de desempleo. En este sentido, se debe dimensionar que una estrategia de reducción de la informalidad necesariamente conlleva un conjunto de acciones orientadas a fomentar las actividades productivas. 
Otro aspecto relevante es que las variaciones de la informalidad dependiente también se explican por factores institucionales como COSTO y SP. En cambio, la informalidad independiente solo por EFICIENCIA. En términos generales se puede argumentar que cualquier esfuerzo por reducir la informalidad implica desde un enfoque institucional, atender los tres factores.

Con relación a la variable COSTO, aumentos en los costos laborales provocan que la informalidad exhiba un crecimiento. Por su parte, SP sugiere que, a mayor cantidad de individuos con acceso a Seguro Popular, mayores serán los niveles de informalidad dependiente observados en el país. Sin embargo, es importante puntualizar que un resultado en esta dirección no debe ser interpretado como un argumento para sostener que el Seguro Popular es un programa social del que debe prescindir la política social. Sus beneficios han sido importantes y además es un programa congruente con las necesidades sociales de quienes están más desprotegidos. Sin embargo, se considera que debería estar diseñado en eliminar posibles inhibidores de los procesos de formalización laboral.

Finalmente, en lo que concierne a la variable de eficiencia en recaudación EFICIENCIA, la mayor eficiencia del gobierno en términos de recaudación se interpone con el objetivo de los individuos por evadir obligaciones formales o en la disminución de sus costos, por lo cual optarán por la informalidad que supone un menor costo. Es decir, para los individuos no existe un incentivo a permanecer formal cuando el gobierno se vuelve más eficiente al recaudar impuestos. Por lo cual la estrategia debería ser resaltar el beneficio de dicha recaudación y que los individuos lo vean reflejado directamente en mejoras de bienestar.

\section{REFERENCIAS BIBLIOGRÁFICAS}

Aguilera, N., y Velázquez, G. (2005). Los efectos de la informalidad. Seguridad Social, (254), 1-7. https://doctrina.vlex.com.mx/vid/efectos-informalidad-329473003 
Busso, M., Fazio, M., y Levy, S. (2012). (In) Formal and (Un) Productive: The Productivity Costs of Excessive Informality in Mexico. IDB Working Paper, 341. https://publications.iadb.org/en/ publication/11062/informal-and-unproductive-productivity-costs-excessive-informality-mexico

Davidson, R., y MacKinnon, J. G. (1993). Estimation and inference in econometrics. Oxford University Press.

Fields, G. S. (2004). Dualism in the labor market: A perspective on the Lewis Model After Half a Century. http://digitalcommons.ilr.cornell.edu/articles/268/

Fields, G. S. (2005). A Guide to Multisector Labor Market Models. Social Protection Discussion Paper Series, (0505), 1-50. http://siteresources.worldbank.org/SOCIALPROTEGTION/Resources/ SP-Discussion-papers/Labor-Market-DP/0505.pdf

Friedman, E., Johnson, S., Kaufmann, D., y Zoido-Lobaton, P. (2000). Dodging the grabbing hand: the determinants of unofficial activity in 69 countries. Fournal of Public Economics, 76(3), 459493. https://doi.org/10.1016/S0047-2727(99)00093-6

Hart, K. (1973). Informal Income Opportunities and Urban Employment in Ghana. The Fournal of Modern African Studies, 11(1), 61-89. https://doi.org/10.1017/S0022278X00008089

Jiménez, D. M. (2012). La informalidad laboral en América Latina: ¿Explicación estructuralista o institucionalista? Cuadernos de Economía, 31(58), 113-143. http://www.scielo.org.co/scielo. php? script=sci_abstract\&pid $=$ S0121-47722012000300006

Jiménez, M. (2013). La informalidad laboral en el sector formal de la Argentina. Un análisis preliminar. Documentos de trabajo, (10), 1-65. https://ri.conicet.gov.ar/handle/11336/3135 
Leal, J. (2014). The informal sector in contemporary models of the aggregate economy. Banco de México Working Papers, (2014-24), 1-20. https://www.banxico.org.mx/publications-and-press/banco-demexico-working-papers/\%7BFA2915B9-8494-C969-A311-F58ACB175938\%7D.pdf

Leontaridi, M. (1998). Segmented Labour Markets: Theory and Evidence. Fournal of Economic Surveys, 12(1). https://doi.org/103-109. 10.1111/1467-6419.00048

Levy, S. (2008). Good Intentions, Bad Outcomes: Social Policy, Informality and Economic Growth in Mexico. Brookings Institution Press.

Loayza, N. (1997). The Economics of the Informal Sector. A Simple Model and Some Empirical Evidence from Latin America. Policy Research Working Paper, 1(1727), 1-60. https://ideas.repec. org/p/wbk/wbrwps/1727.html

Maloney, W., y Saavedra J. (2008). El sector informal: ¿Qué es, por qué nos importa y cómo lo medimos? En G. Perry, W. Maloney, O. Arias, P. Fajnzylber, A. Mason y J. Saavedra. (Ed). Informalidad: Escape y Exclusión (21-41). Banco mundial en coedición con Mayol Ediciones S.A.

McConnell, G., Brue, S., y Macpherson, D. (2003). Economía Laboral. McGraw-Hill.

Moreno, J. (2007). Los salarios del sector formal e informal en México: análisis de ganancias y pérdidas por formalización. Ensayos, 26(1),1-44. https://www.researchgate.net/publication/227430742 Los_salarios_del_sector_formal_e_informal_en_Mexico_analisis_de_ganancias_y_perdidas_ por_formalizacion

Negrete, R., y Eternod, M. (2011). Informalidad y Género. En XII Encuentro Internacional de Estadísticas de Género: empoderamiento, autonomía económica y políticas públicas. Instituto Nacional de Estadística y Geografia (INEGI). 
Organización Internacional del Trabajo (OIT). (2012). Guía de recursos sobre economía informal. http://ilo.org/public/spanish/support/lib/resource/subject/informal.htm

Organización para la Cooperación y el Desarrollo Económicos (OGDE). (2004). Perspectivas del Empleo de la OCDE. Publicaciones de la OCDE.

Packard, T. (2007). Do Workers in Chile Choose Informal Employment? A Dynamic Analysis of Sector Choice. Policy Research Working Paper, (4232), 1-56. https://www.academia.edu/28941129/ Do_workers_in_Chile_choose_informal_employment_A_dynamic_analysis_of_sector_choice

Santana, A. (Goord.). (2011). El Seguro Popular de Salud en México: Posibles efectos sobre la economía informal. Avance-Análisis, Investigación y Estudios para el Desarrollo A.C. https://ethos.org.mx/wpcontent/uploads/2016/04/El-Seguro-Popular-de-Salud-en-Mexico.pdf

Singh, A., Jain-Ghandra, S., y Mohommad, A. (2012). Inclusive Growth, Institutions, and the Underground Economy. IMF Working Paper, (WP/12/47), 1-13.

Tannuri-Pianto, M., y Pianto, D. M. (2002). Informal Employment in Brazil- A Choice at the Top and Segmentation at the Bottom: A Quantile Regression Approach. En XXIV Brazilian Econometrics Meeting, Rio de Janeiro, Brasil. http://citeseerx.ist.psu.edu/viewdoc/ download?doi=10.1.1.454.4705\&rep=repl\&type=pdf

Uribe, J. I., y Ortiz, C. H. (2004). Una propuesta de conceptualización y medición del sector informal. Documentos de trabajo, Universidad del Valle, Departamento de Economía, Centro de Investigaciones y Documentación Socioeconómica. http://bibliotecavirtual.clacso.org.ar/ar/libros/colombia/ cidse/doc76.pdf 
